\title{
MAPK-Hemmung eröffnet neue Perspektiven in der Therapie von Neurofibromen
}

Fragestellung und Hintergrund: Lässt sich mit einer Hemmung des MAPK-Signalwegs das Wachstum von Neurofibromen bei Neurofibromatose Typ 1 hemmen?

Die Neurofibromatose Typ 1 ist eine relativ häufige genetische Erkrankung mit verschiedenen Manifestationsformen, insbesondere am Nervensystem. Namensgebend sind plexiforme Neurofibrome, die bei bis zur Hälfte der Patienten auftreten. Diese Neurofibrome können schmerzhaft sein, neurologische Defizite verursachen und verunstalten. Seltener kommt es zu einer malignen Transformation bis hin $\mathrm{zu}$ einem aggressiv metastasierenden Tumor. Die chirurgische Behandlung ist problematisch, die Strahlentherapie zeigt nur eine begrenzte Wirksamkeit und medikamentöse Therapieoptionen fehlen bisher.

Die der Neurofibromatose zugrundeliegende genetische Störung führt sekundär zu einer Aktivierung des Pfades über RAS und mitogenaktivierte Proteinkinasen (MAPK). Folgerichtig wurde in der vorliegenden Phase-I-Studie ein oraler Hemmstoff der MAPKKinasen (MEK1 und 2) bezüglich seiner Wirksamkeit geprüft.

\section{Originalie}

Dombi E et al. Activity of selumetinib in neurofibromatosis type 1-related plexiform neurofibromas. N Engl J Med. 2016;375(26):2550-60.
Patienten und Methodik: Im Rahmen einer Phase-I-Studie wurden 24 pädiatrische Patienten mit einem medianen Alter von 10,9 Jahren (Bereich 3-18,5 Jahre) mit Selumetinib (AZD6244, ARRY142886) behandelt. Die Sub- stanz wurde zweimal täglich in einer Dosierung von $20-30 \mathrm{mg} /$ $\mathrm{m}^{2}$ Körperoberfläche (KOF) kontinuierlich gegeben. Das Ansprechen wurde mittels Magnetresonanztomografie (MRT) geprüft.

Ergänzend wurde die Substanz auch in einem Mausmodell der Neurofibromatose Typ 1 getestet (DhhCre;Nf1fl/fl).

Ergebnisse: Die Tumoren der pädiatrischen Patienten hatten ein medianes Volumen von $1.205 \mathrm{ml}$ (Bereich: 29-8.744 ml). Median wurden 30 Zyklen (= 30 Monate; Bereich: 6-56 Monate) verabreicht. Die maximal tolerierte Dosis (MTD) lag bei 25 $\mathrm{mg} / \mathrm{m}^{2}$ KOF.

Die wichtigsten Nebenwirkungen waren Hautveränderungen, gastrointestinale Beschwerden und asymptomatische Erhöhungen der Kreatinkinase.

Partielles Ansprechen - definiert als Tumorschrumpfung um mehr als $20 \%$ - wurde bei 17 der 24 Studienteilnehmer (71\%) beobachtet. Eine Progression der Erkrankung unter der Therapie wurde nicht beobachtet.

Im Tiermodell zeigten 12 von 18 Mäusen (67\%) ein Ansprechen in einem ähnlichen Ausmaß.

Schlussfolgerungen der Autoren: Die Hemmung des Wachstums nicht operabler plexiformer Neurofibrome mit dem MAPK-Hemmer Selumetinib bietet eine neue Perspektive für Kinder, die an einer Neurofibromatose Typ 1 erkrankt sind.

Schlüsselwörter: Neurofibrome - Selumetinib - MAPK

\section{- Kommentar von Michael Weller, Zürich, Schweiz}

\section{"Zielgerichtete Therapie der Neurofibromatose Typ 1"}

Die vorliegende Studie deutet einen Durchbruch in der pharmakologischen Therapie der Neurofibrome im Rahmen der Neurofibromatose Typ 1 an - nach zahlreichen überwiegend frustranen Versuchen, das Wachstum dieser Tumoren pharmakologisch zu hemmen. Der Durchbruch beruht auf der gezielten Suche nach einer Substanz, die nahe am Substrat der Erkrankung ansetzt, nämlich der gesteigerten Aktivierung des RAS-MAPK-Signalwegs. Wenngleich der Erfolgsnachweis sich bisher im Wesentlichen auf bildgebende Befunde bezieht und eine prospektive Erfassung der Rückbildung neurologischer Defizite oder sonstiger Parameter des klinischen Benefits fehlen, kann jedoch kaum ein Zweifel bestehen, dass angesichts des neuroradiologischen Ansprechens ein solcher Benefit für Patienten nachweisbar sein sollte. Obwohl die Verträglichkeit gut war, deuten präklinische Untersuchungen darauf hin, dass alternierende Dosierungsschemata vielleicht auch wirksam sein könnten - und besser verträglich. Somit ist damit zu rechnen, dass die Therapieschemata vielleicht noch nicht endgültig de- finiert sind. Gute Verträglichkeit wäre sehr wichtig bei einer Therapie, die grundsätzlich über Jahre, wenn nicht lebenslang gegeben werden müsste. Langzeituntersuchungen werden auch notwendig sein, um das langfristige Verträglichkeits- und Sicherheitsprofil solcher Substanzen besser abschätzen zu können. Ungeachtet dessen ist der Gruppe um Eva Dombi jedoch eine sehr erfolgreiche Studie gelungen.

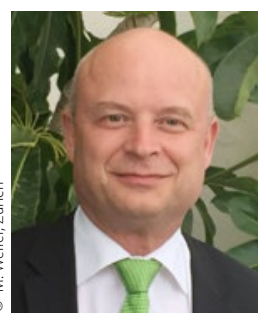

Prof. Dr. Michael Weller

Direktor der Klinik für Neurologie, Universitätsspital Zürich, Schweiz michael.weller@usz.ch 